\title{
Calcified Aneurysms in Coronary Arteries of a 48-Year-Old Patient
}

\author{
Marcelo Kuhn Momolli, José Luis de Castro e Silva Pretto, Daniel Sato, Cristiane Pereira Seibel, \\ Norberto Duda, Roque Paulo Falleiro, Luis Sérgio de Moura Fragomeni
}

Passo Fundo, RS - Brazil

This is a case report of a 48-year-old female patient with a compatible history of Kawasaki disease during childhood, who was admitted to the emergency coronary unit with unstable angina pectoris. Coronary angiography identified two coronary aneurysms, one causing right coronary occlusion and the other causing severe obstruction of the left anterior descending coronary artery. Coronary artery bypass surgery was indicated.

Kawasaki disease is an acute systemic disease, usually self-limited, which affects multiple organs and is found worldwide, however it is more frequent in Asia ${ }^{1,2}$. Kawasaki disease occurs during childhood, usually in children under five years-old, and peaks between the $1^{\text {st }}$ and $2^{\text {nd }}$ years of age ${ }^{2,3}$. The pathogenesis of the disease still remains unknown, but it is suspected that an infectious agent may be involved ${ }^{1,4}$. A staphylococcus toxin may act as a "superantigen" which interacts with T-cells. The diagnosis is made on clinical basis, by exclusion of other possible causes. It is necessary that, besides high fever lasting five or more days, the patient has four of the five following symptoms: nonsuppurative bilateral conjunctivitis, alteration of oral mucus membrane, cervical lymphoadenopathy, exanthema maculo-papular and alterations of the limbs, such as edema and erythema of the palms of hands and feet ${ }^{2,4}$.

The greater morbidity and mortality associated with this condition is linked to the development of cardiovascular complications, such as aneurysms of the coronary arteries, thrombosis, and myocarditis ${ }^{1,4-6}$. Other complications include arthritis, hepatitis, pancreatitis, urethritis, aseptic meningitis, and valvar dysfunction, but aneurysms of the medium caliber peripheral arteries are rare ${ }^{6}$. Management of this disease with non-stenoidal antiinflammatory drues, antithrombotics and immunoglobulin seem to reduce

Faculdade de Medicina da Universidade de Passo Fundo

Mailing address: Luis Sérgio de Moura Fragomeni - Rua Teixeira Soares, 777 S/702 - 99010-080 - Passo fundo, RS - Brasil. E-mail: fragomeni@ annex.com.br cardiovascular risks ${ }^{1,2,4}$. Kawasaki disease should be included in the differential diagnosis of coronary artery diseases, especially in young patients or those without risk factors. The objective of this report is to present the case of a patient with unstable angina due to coronary artery aneurysms, probably as a consequence of Kawasaki disease, who underwent coronary artery bypass surgery.

\section{Case Report}

The patient is a female from the State of Rio Grande do Sul who was hospitalized with complaints of increasing typical angina that started a year earlier. In the previous few months, she had several admitions in the emergency room, when her precordial pain was attributed to emotional factors. She reported that symptoms had worsened in the last few months when she started to have pain at rest. The patient had been a 10-cigarette-a-day smoker for approximately 20 years. On physical examination no alterations were found besides an audible late ystolic click at the $5^{\text {th }}$ left intercostal space. The laboratory investigation and electrocardiography at rest proved to be normal. The chest $\mathrm{X}$-ray showed mediastinal calcifications (fig.1-1). A transthoracic echocardiogram found a hypo-echogenic mass, localized externally to the anterior-lateral wall of the right ventricle, suggesting calcified coronary aneurysm. The mitral valve had systolic prolapse of the leaflets and minimal regurgitation. The other remaining valves and heart chambers were normal, with preserved systolic performance. The coronary angiography diagnosis was proximal occlusion of the right coronary portion of the anterior descending coronary artery, a consequence of compression of these arteries by calcified aneurysms. The anterior descending artery had $90 \%$ stenosis and the right coronary artery was occluded but with adequate collateral flow (figs 1-B and 1-C). During our investigation, the mother reported that the patient at seven years of age had had an illness characterized by fever lasting for two weeks that had been accompanied by pharyngitis, erythema and edema, and 

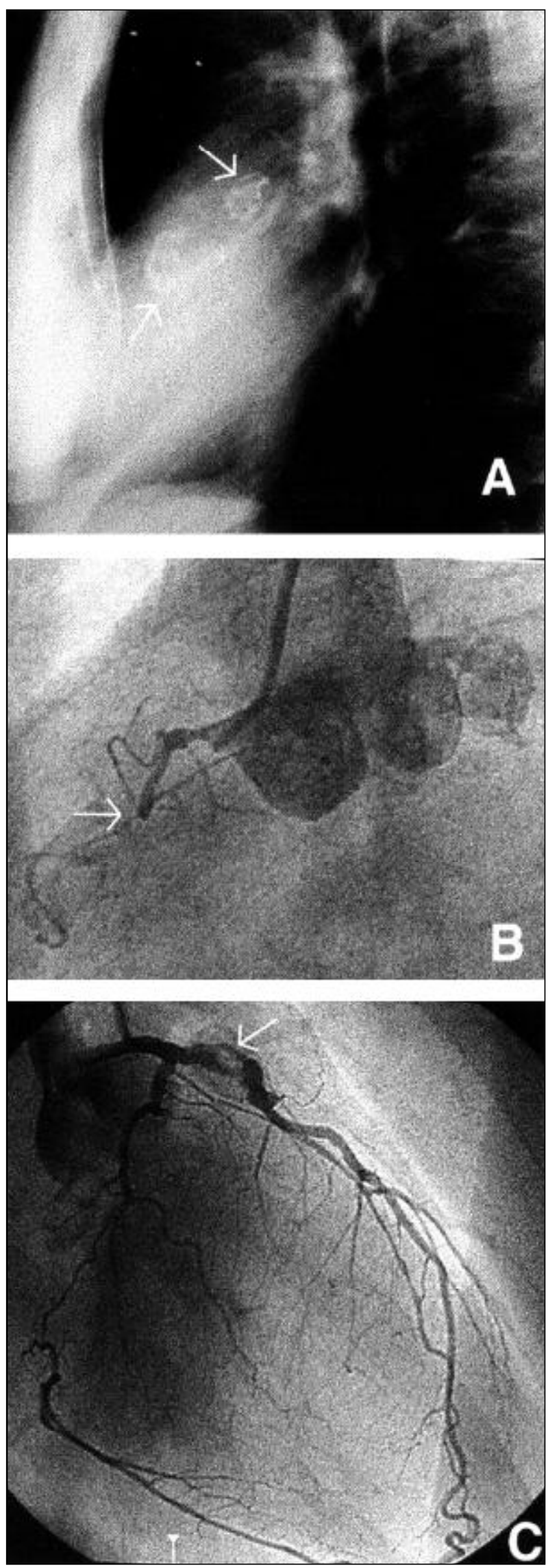

Fig. 1 - A) Chest X-ray demonstrating calcified coronary aneurysms.(arrow); B) oclusion of right coronary (arrow); C) aneurysm and obstruction of anterior descending coronary (arrow). cervical adenopathy. At that time, no diagnosis was established, and after three weeks of hospitalization, patient was discharged in overall improved condition. Based on the clinical evidence, complementary examinations, and the exclusion of other causes, a diagnosis of coronary artery aneurysms, a consequence to Kawasaki disease, was accepted as most probable. With the diagnosis of unstable angina supported by the anatomic findings of the coronary arteries, surgical revascularization of the myocardium was indicated. During surgery the existence of aneurysms of the coronary arteries was confirmed as being responsible for lesions in these vessels (fig.2). With the purpose of reestablishing coronary flow, two vascular grafts were made, for the right coronary artery, an aortocoronary graft with saphenous vein was used, and for the anterior descending artery, the internal thoracic artery was used. All visible tunica intima had signs consistent with arteritis, different, apart from the usual arteriosclerosis patterns, as the anterior descending was stenosed and had an intramyocardial portion. The patient evolved well postsurgery and was discharged on the $7^{\text {th }}$ day.

\section{Discussion}

Kawasaki disease causes an acute inflammation of the tunica intima layer of the arterial vessels, followed by a panvasculitis that may damage coronary arteries predisposed to formation of thrombus or progressive arteriosclerosis ${ }^{3}$. The disease may be clinically silent, with the diagnosis made years or decades later. The frequency of acute cardiovascular complications varies from $30-60 \%$, aneurysms of coronary arteries and thrombosis being the most frequent. At a later stage, persistence of structural alterations may result in manifestation of coronary insufficiency or, in some cases, even sudden death ${ }^{3}$. Approxi-

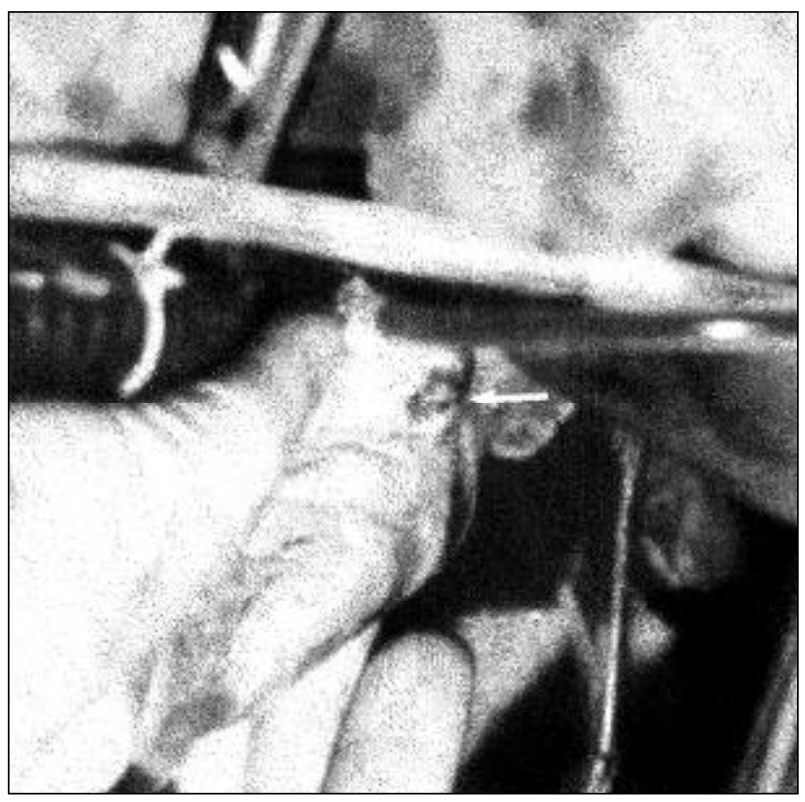

Fig. 2 - Aneurysm of anterior descending coronary is seen at surgery (arrow). 
mately $10-25 \%$ of children affected by Kawasaki disease develop coronary artery aneurysms ${ }^{4-9}$. The natural history of these aneurysms demonstrates that the majority regress in time. Age under one year, female gender, fusiform aneurysm, and 4-mm maximum diameter are factors that indicate a high probability of regression ${ }^{4,6,10}$. Necropsy studies in children with Kawasaki disease revealed that the coronary artery aneurisms may be unique or more rarely, multiple, and that their dimensions vary from $2-8 \mathrm{~mm}$ in their maximum diameter ${ }^{8}$. Studies reveal that the average age of presentation of cardiac findings and presumable sequels of Kawasaki disease is approximately 25 years of age ${ }^{3}$.

The majority of symptoms are brought on by exercise and include chest pain primarily $(60 \%)$ followed by sudden death (16\%) and arrhythmia (10\%). An unusual finding is the X-ray visualization of aneurysms of calcified coronary arteries, which may be useful for the diagnosis, especially in patients with a history compatible with Kawasaki disease, as in this present case ${ }^{3}$. Echocardiography is always indicated when disease is suspected, even when the diagnostic criteria are incomplete. Hiraishi et al. ${ }^{11}$ when analyzing 60 patients with Kawasaki disease obtained, respectively, 95 and $99 \%$ of sensitivity and specificity in the precise identification of aneurysms. The diagnosis of aneurysms of coronary arteries and of obstructive lesions should also be evaluated by coronary angiography after the evidence obtained by clinical data and complemented by electrocardiography, myocardial scintigraphy and echocardiography. In most reports of aneurysms of coronary arteries, the patient does not have a consistent history of Kawasaki disease, and the diagnosis is based on presumed data, after research and exclusion of other condition capable of causing an aneurysm ${ }^{5}$. In this present case, the patient presented with evolving clinical angina, due to severe stenosis of the anterior descending coronary artery and to total obstruction of right coronary artery. For ischemic clinical cases likely the one under discussion, surgical treatment is the most frequently indicated. In select cases, surgical intervention and placement of stents may be an adequate treatment. However, large calcified aneurysms and occluded vessels should undergo revascularization with arterial or venous grafts. Simple resection and terminoterminal- anastomosis after mobilization of right coronary artery is a technical option and has been successfully carried out by Westaby et al. ${ }^{12}$ in two cases of large aneurysms. Currently, the grafts preferentially used in bypass surgery are the arterial ones. In this case, the right coronary could have been revascularized with the right internal thoracic artery had the blood flow not been insufficient. Surgical experience in adults with secondary coronary lesions secondary to Kawasaki disease have rarely been reported, but on the whole show satisfactory results ${ }^{7}$. Attention is called to the fact that due to the nonspecificity of the clinical facts, early diagnosis and adequate management in the acute phase is difficult. However, delay in diagnosis and treatment, which happens more frequently in older children, are linked to a greater incidence of coronary aneurysms. A thorough differential diagnosis and treatment with gamma globulin in the acute phase of the disease can drastically reduce the incidence of later cardiac complications.

\section{References}

1. Laupland KB, Dele DH. Epidemiology, etiology, and management of Kawasaki disease: State of the art. Pediatr Cardiol 1999; 3: 117-83.

2. Rowe RD, Rose V. Kawasaki disease: Canadian update. Can Med Assoc J 1985 ; 1: $25-8$.

3. Burns JC, Shike H, Gordon JB, et al. Sequelae of Kawasaki disease in adolescents and young adults. J Am Coll Cardiol 1996; 1: 253-7.

4. Barros KS. Kawasaki disease. Epidemiology, late prognosis, and therapy. Rheum Dis Clin North Am 1991; 4: 907-19.

5. Shaukat N, Ashraf S, Mebewu A, Freemont A, Keenan D. Myocardial infarction in a young adult due to Kawasaki disease. A case report and review of the late cardiological sequelae of Kawasaki disease. Int J Cardiol 1993; 3: 222-6.

6. Kato H, Sugimura T, Akagi T, et al. Long-term consequences of Kawasaki disease. A 10-to 21-years follow-up study of 594 patients. Circulation 1996; 6: 1379-85.

7. Sato T, Isomura T, Hayashida N, Aoygi S. Coronary artery revascularization in an adult with coronary aneurysms probably secondary to childhood Kawasaki disease. Eur J Cardiothorac Surg 1997; 2: 312-4.

8. Fujiwara T, fujiwara H, Hamashima Y. Frequency and size of coronary arterial aneurysm at necropsy in Kawasaki disease. Am J Cardiol 1987; 8: 808-11.

9. Kato H, Ichimose E, Yoshioka F, et al. Fate of coronary aneurysms in Kawasaki disease: Serial coronary angiography and long-term follow-up study. Am J Cardiol 1982; 49: 1758 .

10. Takahashi M, Mason W, Lewis AB. Regression of coronary aneurysms in patients with Kawasaki syndrome. Circulation 1987; 75: 387.

11. Hiraishi S, Misawa H, Takeda N, et al. Transthoracic ultrasonic visualization of coronary aneurysm, stenosis and occlusion in Kawasaki disease. Heart 2000; 83: 400-5.

12. Westaby S, Vaccari G, Katsumata T. Direct repair of giant right coronary aneurysm. Ann Thorac Surg 1999; 68:1401-03. 